\title{
FINITE PROJECTIVITY AND CONTRAVARIANT FINITENESS
}

\author{
K. IGUSA, S. O. SMALØ, AND G. TODOROV
}

(Communicated by Maurice Auslander)

\begin{abstract}
Let $\Lambda$ be an artin algebra, let $\bmod \Lambda$ be the category of finitely generated $\Lambda$-modules, and let $p d_{1} \bmod \Lambda$ be the subcategory of $\bmod \Lambda$ consisting of the modules of projective dimension less than or equal to one. In this paper it is proved that if the projective dimension of the injective envelope of $\Lambda$ as a $\Lambda$-module is less than or equal to one, then $p d_{1} \bmod \Lambda$ is contravariantly finite in $\bmod \Lambda$. It also contains an example of finitistic projective dimension one where $p d_{1} \bmod \Lambda$ is not contravariantly finite in $\bmod \Lambda$.
\end{abstract}

In studying the category of representations of mathematical structures of different types, one often ends up studying some subcategory of the category of finitely generated modules over an artin algebra. It is therefore important to get more information about such subcategories in general. A natural class of full subcategories to study is those that are closed with respect to direct summands and extensions. Here the question of existence of almost split sequences is essential, and in that connection, the notions of covariant and contravariant finiteness are natural. Let $\Lambda$ be an artin algebra and let $\bmod \Lambda$ be the category of finitely generated $\Lambda$-modules. This note will deal with the full subcategories $p d_{n}$ mod $\Lambda$ consisting of those modules $M$ that have projective dimension less than or equal to $n$, and $f p d \bmod \Lambda$, the full subcategory of $\bmod \Lambda$ consisting of all modules of finite projective dimension. These subcategories are obviously closed with respect to summands and extensions.

The main aim of this paper is to shed some light on the question of when $p d_{n} \bmod \Lambda$ is contravariantly finite in $\bmod \Lambda$. More precisely, we first prove that if the projective dimension of the injective envelope of $\Lambda$ as a left $\Lambda$ module is less than or equal to one, then $p d_{1} \bmod \Lambda$ is contravariantly finite in $\bmod \Lambda$ (see $\S 1$ for the definitions involved). Combining this with the result of Auslander and Reiten [AR] which states that $p d_{1} \bmod \Lambda$ is always covariantly finite in $\bmod \Lambda$, we obtain, in this case, that $p d_{1} \bmod \Lambda$ is then functorially finite in $\bmod \Lambda$. Next, we include an interesting example showing that $f p d$ $\bmod \Lambda$ is not always contravariantly finite in $\bmod \Lambda$. In fact, the finitistic projective dimension of the example is one, so $f p d \bmod \Lambda$ is equal to $p d_{n}$ $\bmod \Lambda$ for all $n$ greater than or equal to one.

Received by the editors October 5, 1989 and, in revised form, November 28, 1989.

1980 Mathematics Subject Classification (1985 Revision). Primary 16A64, 16A60, 16A46, $18 \mathrm{G} 20$. 


\section{Preliminaries}

This section is devoted to recalling the notions and definitions needed in the rest of the paper and to fixing the notation.

Let $\Lambda$ be an artin algebra, $\bmod \Lambda$ the category of finitely generated left $\Lambda$-modules, $p d_{n} \bmod \Lambda$ the full subcategory of $\bmod \Lambda$ consisting of those modules of projective dimension less than or equal to $n$, and $f p d \bmod \Lambda$ the full subcategory of $\bmod \Lambda$ consisting of all modules of finite projective dimension.

Recall that a covariant functor $F$ from a category $\mathscr{A}$ to the category of sets is said to be finitely generated if there exist an object $A$ in $\mathscr{A}$ and a natural transformation from $\mathscr{A}(A$,$) onto F$. The definition of a finitely generated contravariant functor is analogous.

From [AS1] we have the following definition.

Definition. A full subcategory $\mathscr{A}$ of a category $\mathscr{B}$ is said to be

(i) contravariantly finite in $\mathscr{B}$ if for each object $X$ in $\mathscr{B}$, the representable functor $\mathscr{B}(, X)$ restricted to $\mathscr{A}$ is finitely generated as a functor on $\mathscr{A}$,

(ii) covariantly finite in $\mathscr{B}$ if for each object $X$ in $\mathscr{B}$, the representable functor $\mathscr{B}(X$,$) restricted to \mathscr{A}$ is finitely generated, and

(iii) functorially finite in $\mathscr{B}$ if it is both contravariantly and covariantly finite in $\mathscr{B}$.

\section{THE RESULTS}

This section is devoted to giving the example with the properties explained in the introduction and to proving the following result.

Theorem 2.1. If the projective dimension of the injective envelope of $\Lambda$ as a left $\Lambda$-module is less than or equal to one then $p d_{1} \bmod \Lambda$ is contravariantly finite in $\bmod \Lambda$.

Proof. Let $\Lambda$ be an artin algebra such that $I_{0}(\Lambda)$, the injective envelope of $\Lambda$ as a left $\Lambda$-module, has projective dimension less than or equal to one. Let $X$ be any finitely generated left $\Lambda$-module and let $P_{1} \stackrel{f_{1}}{\longrightarrow} P_{0} \stackrel{f_{0}}{\longrightarrow} X \rightarrow 0$ be a projective presentation of $X$. Let $0 \rightarrow P_{1} \stackrel{g_{1}}{\longrightarrow} I_{0}$ be an injective envelope of $P_{1}$ and consider the following diagram where id denotes the identity and $h$ is the induced morphism.

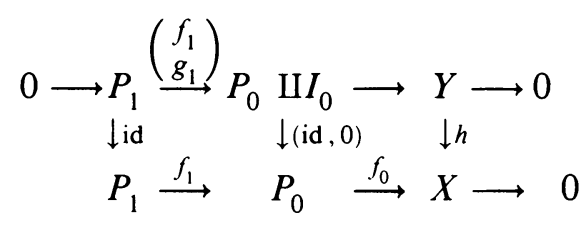

One sees immediately that this diagram commutes. Further, $h$ is an epimorphism, $Y$ has projective dimension less than or equal to one, and $K$, the kernel 
of $h$, is a quotient of the injective module $I_{0}$. We now claim that

$$
\operatorname{Hom}_{\Lambda}(, h):\left.\left.\operatorname{Hom}_{\Lambda}(, Y)\right|_{p d_{1} \bmod \Lambda} \rightarrow \operatorname{Hom}_{\Lambda}(, X)\right|_{p d_{1} \bmod \Lambda}
$$

is an epimorphism. To prove this, let $Z$ be a module of projective dimension less than or equal to one. Then $\operatorname{Ext}^{1}(Z$,$) is right exact, and therefore vanishes$ on quotients of injective modules. Hence, $\operatorname{Ext}^{1}(Z, K)$ is zero for each module $Z$ of projective dimension less than or equal to one. Therefore we obtain that the following sequence is exact for each module $Z$ of projective dimension less than or equal to one.

$$
0 \rightarrow \operatorname{Hom}_{\Lambda}(Z, K) \rightarrow \operatorname{Hom}_{\Lambda}(Z, Y) \rightarrow \operatorname{Hom}_{\Lambda}(Z, X) \rightarrow 0 .
$$

This completes the proof of the claim as well as the proof of the whole theorem.

Combining this result with results from [AR, AS2] we obtain the following corollary.

Corollary 2.2. If the projective dimension of the injective envelope of $\Lambda$ as a left $\Lambda$-module is less than or equal to one, then for each indecomposable module $X$ in $p d_{1} \bmod \Lambda$ the following hold true:

(i) there is a module $Y$ in $p d_{1} \bmod \Lambda$ and a left almost split morphism $f$ from $X$ to $Y$,

(ii) there is module $Z$ in $p d_{1} \bmod \Lambda$ and a right almost split morphism $g$ from $Z$ to $X$.

In particular, $p d_{1} \bmod \Lambda$ has almost split sequences.

Proof. We have from the theorem that $p d_{1} \bmod \Lambda$ is contravariantly finite in $\bmod \Lambda$ and from $[\mathrm{AR}]$ we have that $p d_{1} \bmod \Lambda$ is covariantly finite in $\bmod \Lambda$ for all artin algebras $\Lambda$, hence $p d_{1} \bmod \Lambda$ is functorially finite in $\bmod \Lambda$. Therefore we can apply Theorem 2.4 of [AS2] stating that any functorially finite subcategory of $\bmod \Lambda$ that is closed under summands and extension has almost split sequences.

We now give the example of an artin algebra such that $f p d \bmod \Lambda$ is not contravariantly finite in $\bmod \Lambda$.

Let $k$ be an algebraically closed field and let $\Lambda$ be given as the path algebra of the quiver

$$
1 \cdot \frac{\stackrel{\beta}{\alpha}}{\stackrel{\gamma}{\longrightarrow}} \cdot 2
$$

modulo the ideal generated by the paths $\alpha \gamma, \beta \gamma$, and $\gamma \alpha$, where $\rho \sigma$ means first the path $\sigma$ followed by the path $\rho$.

Proposition 2.3. Let $\Lambda$ be the algebra described above. Then $p d_{1} \bmod \Lambda$ is equal to $f p d \bmod \Lambda$ and $p d_{1} \bmod \Lambda$ is not contravariantly finite in $\bmod \Lambda$.

In the proof of this we need the following easily verified observation about submodules of modules which is the direct sum of a semisimple module and a projective module of Loewy length equal to two. 
Lemma 2.4. Let $M$ be the direct sum of a semisimple module and a projective module of Loewy length two. Then every submodule of $M$ is a direct sum of a semisimple module and a projective module.

Proof of the proposition. To prove that the example presented above has the claimed properties, we first observe that the two nonisomorphic indecomposable projective modules that we denote $P_{1}$ and $P_{2}$, according to the enumeration in the quiver, have dimension two and four as $k$-vector spaces, respectively. Therefore each $\Lambda$-module of odd $k$-dimension has infinite projective dimension. Hence the simple $\Lambda$-modules, that are one-dimensional as $k$-vector spaces, have infinite projective dimension. Further, $P_{1}$ has only one proper submodule that is then simple, and each indecomposable proper submodule of $P_{2}$ is either isomorphic to $P_{1}$ or is simple. Since $P_{1}$ has length equal to two, this implies by Lemma 2.4 that each submodule of the radical of a projective module is isomorphic to a direct sum of a semisimple module and the direct sum of copies of $P_{1}$. From this we deduce that any $\Lambda$-module has either infinite projective dimension or projective dimension less than or equal to one. Hence, for this $\Lambda, f p d \bmod \Lambda$ is equal to $p d_{1} \bmod \Lambda$.

We now prove that $p d_{1} \bmod \Lambda$ is not contravariantly finite in $\bmod \Lambda$. Consider the simple $\Lambda$-module $S_{2}=P_{2} / \operatorname{rad} P_{2}$, where rad denotes the radical. This module is the simple module corresponding to the vertex 2 in the quiver. We prove that there is no finitely generated $\Lambda$-module $X$ of projective dimension one with a $\Lambda$-homomorphism $f$ from $X$ to $S_{2}$ such that

$$
\left.\operatorname{Hom}_{\Lambda}(, f)\right|_{p d_{1} \bmod \Lambda}:\left.\left.\operatorname{Hom}(, X)\right|_{p d_{1} \bmod \Lambda} \rightarrow \operatorname{Hom}\left(, S_{2}\right)\right|_{p d_{1} \bmod \Lambda}
$$

is surjective. Assume there exists such an $X$ and $f$. Choose one such $X$ of minimal length and also call the corresponding $\Lambda$-homomorphism $f$. We now claim that $X$ does not contain any submodule isomorphic to $P_{1}$. For if $X$ contains a submodule isomorphic to $P_{1}$, this submodule could be divided out, thus producing a quotient module $X^{\prime}$ of less length than $X$, of projective dimension one and, since $\operatorname{Hom}\left(P_{1}, S_{2}\right)$ is zero, $f$ would induce a homomorphism $f^{\prime}$ from $X^{\prime}$ to $S_{2}$. Therefore,

$$
\left.\operatorname{Hom}_{\Lambda}\left(, f^{\prime}\right)\right|_{p d_{1} \bmod \Lambda}:\left.\left.\operatorname{Hom}\left(, X^{\prime}\right)\right|_{p d_{1} \bmod \Lambda} \rightarrow \operatorname{Hom}\left(, S_{2}\right)\right|_{p d_{1} \bmod \Lambda}
$$

would also be surjective, contradicting the minimality of the length of $X$. Hence, $X$ is annihilated by the element $\gamma$, and, therefore, $X$ is a $\Lambda /(\gamma)$ module, where $(\gamma)$ is the ideal generated by $\gamma$. We are, therefore, in the situation where we are considering modules over the "double arrow", that was classified almost a hundred years ago by Kronecker [K] (see [DR] for details). There is a family of indecomposable $\Lambda$-modules, $\left\{Y_{i}\right\}$, of length two, of projective dimension one, annihilated by $\gamma$, indexed over the projective line of the field $k$ (excluding one point) with a nonzero $\Lambda$-homomorphism to $S_{2}$. Using the classification of the indecomposable representations of the "double arrow", we know from [DR] that the only indecomposable finitely generated 
$\Lambda$-modules annihilated by $\gamma$ with a nonzero $\Lambda$-homomorphism from any two of the modules in the family $\left\{Y_{i}\right\}$ of indecomposable modules, are the preinjective $\Lambda /(\gamma)$-modules. Hence, $X$ has to have an indecomposable preinjective $\Lambda /(\gamma)$-module as a direct summand. However, each indecomposable preinjective $\Lambda /(\gamma)$-module has odd $k$-dimension and, therefore, has infinite projective dimension as a $\Lambda$-module. This contradicts the existence of $X$ and $f$, and completes the proof of the proposition.

\section{REFERENCES}

[AS1] M. Auslander and S. O. Smalø, Preprojective modules over Artin algebras, J. Algebra 66 (1980), 61-122.

[AS2] _ Almost split sequences in subcategories, J. Algebra 69 (1981), 426-454.

[AR] M. Auslander and I. Reiten, Applications of contravariantly finite subcategories, Adv. in Math. (to appear).

[DR] V. Dlab and C. M. Ringel, The representations of tame hereditary Artin algebras, Lecture Notes in Pure and Appl. Math., vol. 37, Dekker, New York, 1978.

[K] L. Kronecker, Algebraische Reduction der Scharen bilinearer Formen, Sitzungsber. Sächs. Akad. Wiss. Leipzig. Math. Natur. Kl. Berlin, 1890, pp. 1225-1237.

[S] S. O. Smalø, Functorial finite subcategories over triangular matrix rings, Proc. Amer. Math. Soc. (to appear).

Department of Mathematics, Brandeis University, Waltham, Massachusetts 02254

Institutt for Matematikk og Statistikk, Universitetet I Trondheim, AVH, N-7055 DragVoll, Norway

Department of Mathematics, Northeastern University, Boston, Massachusetts 02115 